\title{
Development of an Electrostatic Precipitator to Remove Martian Atmospheric Dust from ISRU Gas Intakes during Planetary Exploration Missions
}

\author{
J. Sid Clements \\ Member, IEEE \\ Appalachian State University \\ Dept. of Physics and Astronomy \\ Boone, NC 28608 USA \\ clementsjs@appstate.edu \\ Michael R. Johansen \\ Electrostatics and Surface Physics Lab \\ NASA KSC FL 32899 USA \\ michael.johansen@nasa.gov \\ M. Loraine Lowder \\ Atlanta Metropolitan College \\ Div. of Science, Math and Health Prof. \\ Atlanta, GA 30310 \\ mlowder@atlm.edu
}

\author{
Samuel M. Thompson \\ Appalachian State University \\ Dept. of Physics and Astronomy \\ Boone, NC 28608 USA \\ samuelmaxton@gmail.com
}

Blakeley S. Williams

University of South Alabama

Mobile, AL 36688 USA

blakeley.williams@nasa.gov

\author{
Carlos I. Calle \\ Member, IEEE \\ Electrostatics and Surface Physics Lab \\ NASA KSC FL 32899 USA \\ carlos.i.calle@nasa.gov
}

\author{
Nathan D. Cox \\ Appalachian State University \\ Dept. of Physics and Astronomy \\ Boone, NC 28608 USA \\ coxnd@email.appstate.edu
}

\author{
Michael D. Hogue \\ Electrostatics and Surface Physics Lab \\ NASA KSC FL 32899 USA \\ michael.d.hogue@nasa.góv
}

\begin{abstract}
Manned exploration missions to Mars will need dependable in situ resource utilization (ISRU) for the production of oxygen and other commodities. One of these resources is the Martian atmosphere itself, which is composed of carbon dioxide $(95.3 \%)$, nitrogen $(2.7 \%)$, argon $(1.6 \%)$, oxygen $(0.13 \%)$, carbon monoxide $(0.07 \%)$, and water vapor $(0.03 \%)$, as well as other trace gases. However, the Martian atmosphere also contains relatively large amounts of dust, uploaded by frequent dust devils and high winds. To make this gas usable for oxygen extraction in specialized chambers requires the removal of most of the dust. An electrostatic precipitator (ESP) system is an obvious choice. But with an atmospheric pressure just one-hundredth of Earth's, electrical breakdown at low voltages makes the implementation of the electrostatic precipitator technology very challenging. Ion mobility, drag forces, dust particle charging, and migration velocity are also affected because the low gas pressure results in molecular mean free paths that are approximately one hundred times longer than those at Earth atmospheric pressure. We report here on our efforts to develop this technology at the Kennedy Space Center, using gases with approximately the same composition as the Martian atmosphere in a vacuum chamber at 9 mbars, the atmospheric pressure on Mars. We also present $I-V$ curves and large particle charging data for various versions of wire-cylinder and rod-cylinder geometry ESPs. Preliminary results suggest that use of an ESP for dust collection on Mars may be feasible, but further testing with Martian dust simulant is required.
\end{abstract}

Index Terms-carbon dioxide, corona discharge, electrostatic precipitation (ESP), Martian dust, particle charging.

\footnotetext{
* This project was supported by NASA's ISRU project.
}

\section{INTRODUCTION}

Future human exploration of the planet Mars will require the utilization of local resources to reduce the mass, cost, and risk of space exploration. NASA's In Situ Resource Utilization (ISRU) project is to produce mission consumables, surface construction, manufacturing and repair, and space utilities and power [1]. Oxygen, methane, and water production from Martian atmospheric gas requires the removal of ever present dust particles that are uplifted by frequent dust devils and large dust storms. In this paper, we report on the design and testing of an electrostatic precipitator (ESP) that can remove dust from the atmospheric intakes of ISRU production chambers. An ESP designed for the Martian atmospheric environment must work at gas pressures $\sim 100 \times$ lower than Earth atmospheric pressure with much lower gas breakdown voltages.

There are several choices for this task, the two most obvious being the fabric filter and electrostatic precipitator (ESP) [2]. The fabric filter has a pressure drop and therefore requires upstream compression of the gas to be treated because the ambient pressure on Mars is too close to vacuum to use a vacuum pump downstream of the filter to draw the gas through the filter. However, the abrasive Martian dust could damage the compressor. An electrostatically-enhanced filter may have a low enough pressure drop, therefore that option may also be tested in the future. The electrostatic precipitator was chosen for the current project because it does not suffer from the pressure drop problem. However, the low pressure of the Martian atmosphere, at 7 to 10 mbars, precludes the large voltages normally used in terrestrial electrostatic precipitators: if the applied voltage is increased too much, the corona transitions to a localized glow/streamer discharge unsuitable for ESP operation. 
Therefore almost no studies have been performed on low pressure $\mathrm{CO}_{2}$ ESPs with the exception of Pang et al [3] which contains nice calculations of the pertinent parameters under Martian atmospheric conditions and also reports results for a non-customary ESP-type system using a discharge wire external to a tube or torus to collect dust settling out of the Martian atmosphere onto a solar panel. In this paper, we report on our efforts to design a traditional wire-cylinder electrostatic precipitator [2],[4],[5] modified so that it can sustain a charging corona and collection electric field for the challenging Martian atmospheric environment. We also present I-V curves and large particle charging data for several particle and ESP sizes.

\section{MARTIAN ATMOSPHERE}

\section{A. The dusty atmospheric environment}

The surface of Mars is entirely covered with dust with a similar composition and size throughout the planet due to its constant redistribution during global dust storms and localized dust devils. These dust storms and dust devils uplift dust into the rarefied atmosphere (

Fig 1). Calculations based on optical instrumentation on spacecraft in orbit around Mars and on rovers on the surface have yielded estimates for the average diameter of dust particles in the atmosphere. Using this data, Landis et al believe that Martian atmospheric dust has a three-component particle size distribution: atmospheric dust suspended for long periods of time, with diameter ranging from 2 to $4 \mu \mathrm{m}$, with an average value of $3 \pm 0.4 \mu \mathrm{m}$; settled dust, raised into the atmosphere by wind and dust devils, with a diameter under $10 \mu \mathrm{m}$; and saltating particles greater than $80 \mu \mathrm{m}$ [6].

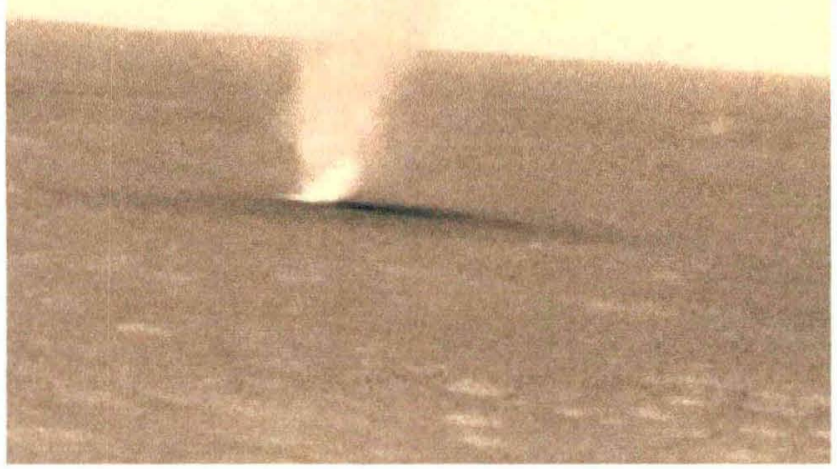

Fig 1. A Martian dust devil photographed by the Spirit rover, $C$ NASA, reprinted with permission.

\section{B. Electrical properties of the Martian atmosphere}

Atmospheric dust removal with an electrostatic precipitator requires the establishment of a large electric field for particle charging and collection. However, the low pressure of the Martian atmosphere greatly limits the potentials that can be applied to generate the field. At 7 to
10 mbars atmospheric pressure, Paschen breakdown occurs at relatively low potentials [7], [8]. Paschen's law states that breakdown voltage for a uniform electric field depends on the product of gap length $d$ and gas number density. Historically, the gas pressure $p$ at standard temperature is plotted instead of number density, but these curves must be adjusted for the low temperature regions on Mars. Breakdown experiments with uniform fields have been performed for most elemental and molecular gases, including $\mathrm{CO}_{2}[9]$. The gas composition affects the electrical properties of the gas. For example, $\mathrm{CO}_{2}$ is electronegative (captures free electrons) and polyatomic (lowers electron swarm energies) [10]. In 2004, we performed experiments with a premixed gas to emulate the Martian atmosphere, composed of a mixture of $95 \% \mathrm{CO}_{2}, 2.7 \% \mathrm{~N}_{2}, 1.6 \% \mathrm{~A}$, $0.13 \% \mathrm{O}_{2}$, and $0.07 \% \mathrm{CO}[11]$. Data was taken at pressures ranging from 270 mbars to 466 mbars (200 mtorr to 350 torr) with gaps of $5 \mathrm{~mm}, 7.5 \mathrm{~mm}$, and $10 \mathrm{~mm}$. The low pressure data (Fig. 2) shows that breakdown potentials in $\mathrm{CO}_{2}$ and in the Martian gas mixture are extremely similar. It appears as if $\mathrm{CO}_{2}$ dictates the Paschen breakdown and that the other gases, present in relatively low concentrations, do not affect it substantially. The Martian gas breakdown voltages are equal to or slightly smaller than the $\mathrm{CO}_{2}$ voltages by an average of 15 volts, perhaps due to the known lower breakdown potentials of nitrogen and argon, present in smaller concentrations. Based on these results, in the present study we used a $95 \% \mathrm{CO}_{2} / 5 \%$ humid air mixture to approximate the Martian atmosphere (contains $0.03 \% \mathrm{H}_{2} 0$ ). For a Mars atmospheric pressure of 7 mbars (5.2 torr), the breakdown potentials that we obtained in a uniform field are $725 \mathrm{~V}$ for a 5 -mm gap, $800 \mathrm{~V}$ for a $7.5-\mathrm{mm}$ gap, and 895 for a 10-mm gap. From our Paschen plot, we expect breakdown on the order of $2.8 \mathrm{kV}$ for a $10-\mathrm{cm}$ gap.

\section{$\mathrm{CO}_{2}$ and Mars Paschen Breakdown}

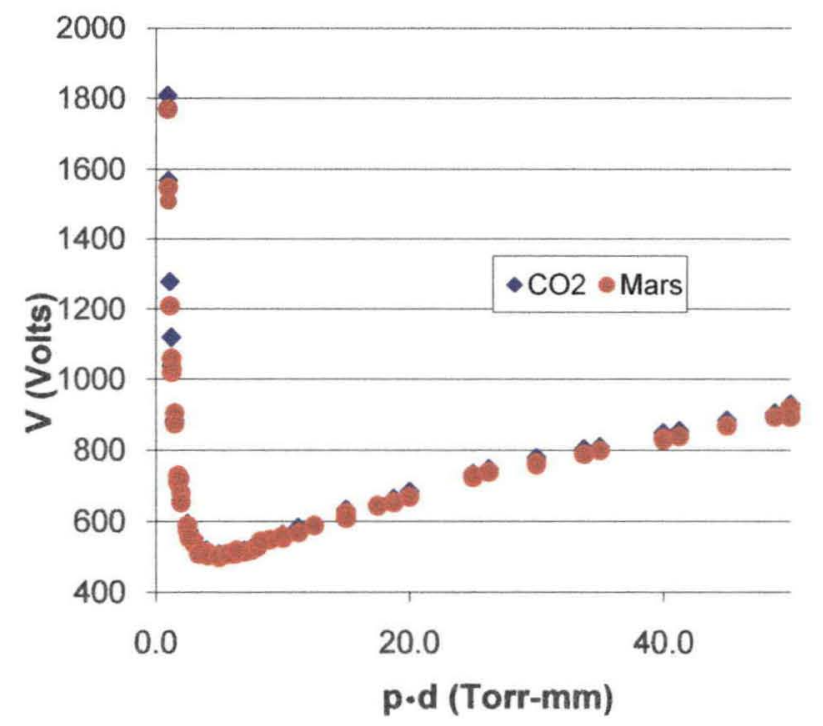

Fig. 2. Paschen breakdown potentials versus pressure-distance for a Martian gas mixture (red circles) and for $\mathrm{CO}_{2}$ (blue triangles) [11]. 
In addition to gas breakdown, dust particle charging and migration is also affected by the low gas pressures on Mars. The roughly $100 \times$ lower gas pressure on Mars results in molecular mean free paths $\lambda$ that are $\sim 100 \times$ longer than at Earth atmospheric pressure, e.g., for $\mathrm{CO}_{2}, \lambda=44 \mathrm{~nm}$ at one atmosphere [12] and $\sim 4 \mu \mathrm{m}$ at 9 mbars. The mean free path can be adjusted for temperature and pressure using the equations in [12] with the Sutherland constant for $\mathrm{CO}_{2}=$ 220. There are three particle charging regimes that are defined based on the Knudsen number $K_{n}$, which is simply the ratio of $\lambda$ to particle radius. The regimes are continuum $\left(K_{n}<<1\right)$, free molecular $\left(K_{n}>1\right)$, and transitional $\left(K_{n} \sim\right.$ 1). The low pressure on Mars shifts dust particle charging from the continuum regime to the transitional. Drag on the particle as it moves through the gas is also affected. Stoke's drag is proportional to the particle radius and the gas viscosity. Although viscosity only depends on temperature and not pressure, Stoke's formula still has to be adjusted using the Cunningham slip correction factor to account for the particle "slipping" between the gas molecules due to their large mean free paths.

\section{EleCtrostatic PrECIPITATOR Physics}

An electrostatic precipitator (ESP) consists of two asymmetric electrodes set at a potential difference. Dust particles are charged using corona generation around the high voltage discharge electrode, which ionizes gas molecules. These ions are accelerated by the electric field $E$ in the region between the electrodes, but numerous collisions with gas molecules results in a fairly constant average drift velocity characterized by an ion mobility $b=$ velocity/E. The ions transfer charge to dust particles encountered in their path as they drift to the grounded collecting electrode. The ions form space charge which modifies the applied $E$ between the electrodes. The electric field for a wire-cylinder geometry without space charge is given by:

$$
E(r)=\frac{V}{r \ln \left(\frac{R}{a}\right)}
$$

where $V$ is the applied voltage, $r$ is the radial distance from the wire, $a$ is the wire radius, and $R$ is the cylinder radius. Ref.[13] contains an equation for the case with space charge:

$$
E(r)=\sqrt{\frac{I}{2 \pi \varepsilon_{0} L b}+\left(\frac{a}{r}\right)^{2}\left\{\left(\frac{V}{a \ln \frac{R}{a}}\right)^{2}-\frac{I}{2 \pi \varepsilon_{0} L b}\right\}}
$$

where $V$ is the applied voltage, $I$ is the ion current, $L$ is the active length of the wire-cylinder, $R$ is the cylinder radius, $a$ is the wire radius, $b$ is the ion mobility and $\varepsilon_{0}$ is the permittivity of free space. Larger ion currents yield more

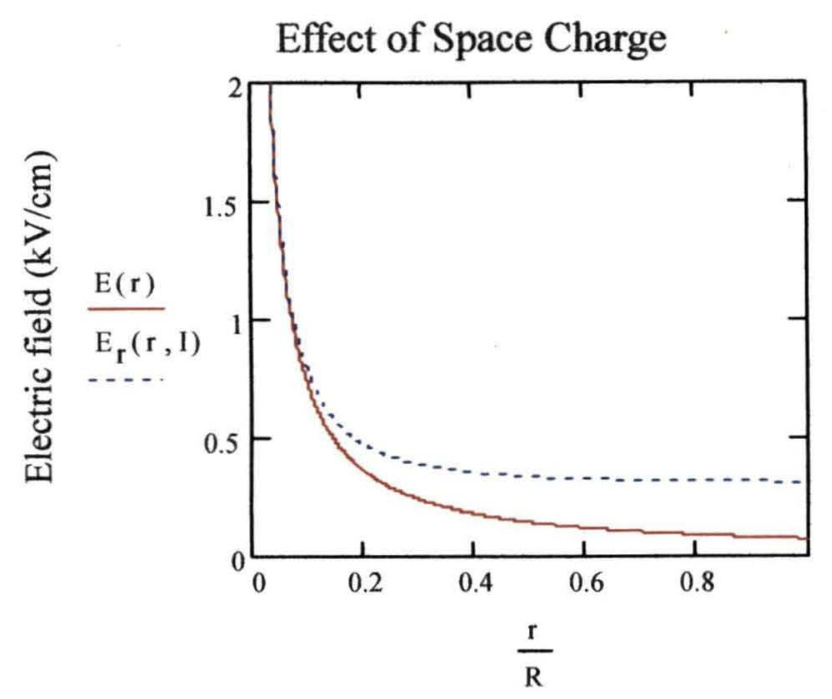

Relative radial position

Fig. 3 Electric fields inside a $7.1-\mathrm{cm}$ diameter cylinder with a $100-\mu \mathrm{m}$ wire as a function of radial distance both without [solid curve using (1)] and with [dashed curve using (2)] space charge. $\mathrm{V}=1.69 \mathrm{kV}$ and $\mathrm{I}=127 \mu \mathrm{A}$

space charge, which increases the electric field substantially, as can be seen in Fig. 3.

Gas ions formed by the strong electric field in a precipitator charge dust particles in two ways: field charging and diffusion charging. In field charging, the drifting ions follow the electric field lines to the dust particles. The number of possible ions attaching to the surface of a particle is clearly proportional to the square of the particle radius. The charging process continues until charge saturation $Q_{s}$ is achieved, which takes place with a characteristic time $\tau$ given by

$$
\tau=\frac{4 \varepsilon_{0}}{N_{0} e b}
$$

where $N_{0}$ is the ion number density in $\mathrm{m}^{-3}, e$ is the fundamental charge, and $b$ is the ion mobility. For positive ions in $\mathrm{CO}_{2}$ at one Earth atmosphere, $b=0.84 \times 10^{-4} \frac{\mathrm{m}^{2}}{V \cdot s}[5]$. The product of mobility and pressure is constant for positive ions in $\mathrm{CO}_{2}$ in the pressure range between Mars pressures and one Earth atmosphere [14]. Therefore, the mobility at Mars pressure should be $b=0.008 \frac{m^{2}}{V \cdot s}$ at 9 mbars. The particle charge as a function of time $q(t)$ is given by:

$$
q(t)=Q_{s} \frac{t}{t+\tau}
$$

The Pauthenier saturation charge $Q_{s}$ for a spherical particle of diameter $d$ is reached when the force on an approaching gas ion due to the field $\mathrm{E}$ and the repulsive force from the charged particle are balanced [15].

Pauthenier charging, also known as field charging, is valid in the continuum regime $\left(K_{n}<<1\right)$ and is given by 


$$
Q_{s}=12 \pi \varepsilon_{0} \frac{k}{k+2}\left(\frac{d}{2}\right)^{2} E
$$

where $k$ is the relative permittivity of the particle. The term $(k / k+2)=1$ for conductive particles.

In diffusion charging, ions diffuse through the gas due to thermal motion, colliding with the dust particles at random. Technically, diffusion charging does not have a saturation limit because the charging does not stop but instead the rate slows as the particle acquires charge which repels the incoming ions. The ions have a distribution of velocities and energies, and ions with too little energy are repelled. Eventually only a very few ions in the high energy tail of the distribution can reach the particle, and the amount of additional charging becomes insignificant. The diffusion charge acquired by dust particles can be calculated using standard kinetic theory of gases. This charge depends on the ion number density, $N_{\theta}$, their mean velocity, $c_{i}$, and the diameter $d$ of the particle. Cross [2] reports the expression developed by Arent [16] in the continuum regime for the diffusion charge $q$ as a function of time $t$

$$
q(t)=\frac{4 \pi \epsilon_{0} k T}{\mathrm{e}} \ln \left(\frac{\frac{d}{2} N_{0} \mathrm{e}^{2} c_{i} t}{4 \epsilon_{0} k T}+1\right)
$$

where $k$ is Boltzmann's constant, and $T$ is the temperature of the gas in Kelvin.

The mean ion velocity $c_{i}$ for $\mathrm{CO}_{2}$ at 9 mbars ( 7 torr) and $273 \mathrm{~K}$ can be calculated from kinetic theory using the following expression [17]

$$
c_{i}=\left(\frac{8 R T}{\pi M}\right)^{\frac{1}{2}}=362 \frac{\mathrm{m}}{\mathrm{s}}
$$

where $R$ is the universal gas constant and $M$ the molecular mass of $\mathrm{CO}_{2}$. The ion number density $N_{0}$ can be calculated using the relationships

$$
\begin{gathered}
N_{0}=\frac{J}{e b E} \\
\mathrm{~J}=\frac{\mathrm{I}}{\mathrm{A}}
\end{gathered}
$$

where $J$ is the current density, $I$ is the measured current flowing from the corona wire to the ground electrode, $A$ is the area the current flows through, and $E$ is from (2).

For example, using (8) and (9) at a $\mathrm{CO}_{2}$ pressure of 9 mbars, the ion number density $N_{0}$ halfway between the electrodes for a 7.1-cm cylinder - $100-\mu \mathrm{m}$ wire ESP at a 1.7 $\mathrm{kV}$ operating voltage and $I=127 \mu \mathrm{A}$ is $N_{0}=8.9 \times 10^{13}$ ions $/ \mathrm{m}^{3}$ [with $b=0.008 \frac{\mathrm{m}^{2}}{V \cdot s}$ and $E=0.35 \mathrm{kV} / \mathrm{cm}$ from (2) and Fig. 3]. Under these typical conditions, field charging dominates for large particle sizes and short exposure times.
Fig. 4 shows both field and diffusion charging for $1-\mu \mathrm{m}$ and $5-\mu \mathrm{m}$ diameter particles as a function of time. For $5-\mu \mathrm{m}$ particles, field charging dominates for times up to $0.1 \mathrm{~s}$, and is still larger than diffusion even at $t=10 \mathrm{~s}$. Diffusion charging dominates for $1-\mu \mathrm{m}$ particles for exposure times greater than $10 \mathrm{~ms}$. The region between requires a theory which incorporates both mechanisms at the same time [18], [19], but simply adding the two is a fair approximation [2]. The diameters of particles in the Martian atmosphere range from 2 to $10 \mu \mathrm{m}$. In this case, both field and diffusion charging contribute to the charging mechanism, with field charging contributing more to the larger 4 to $10 \mu \mathrm{m}$ particles uplifted into the atmosphere during dust storm and dust devil activity, and both mechanisms contributing to the 2 to $4 \mu \mathrm{m}$ particles normally found in the Martian atmosphere. Continuum regime theory only provides an order of magnitude estimate because transitional regime theory is more appropriate for this particle size range at Mars gas pressures. In fact, the longer mean free paths at the low Mars pressures may shift the dominant diffusion charging size range to larger dust particles. Unfortunately, transitional regime theory is quite complicated and several different ones requiring numerical solution have been proposed in the literature [20]-[23].

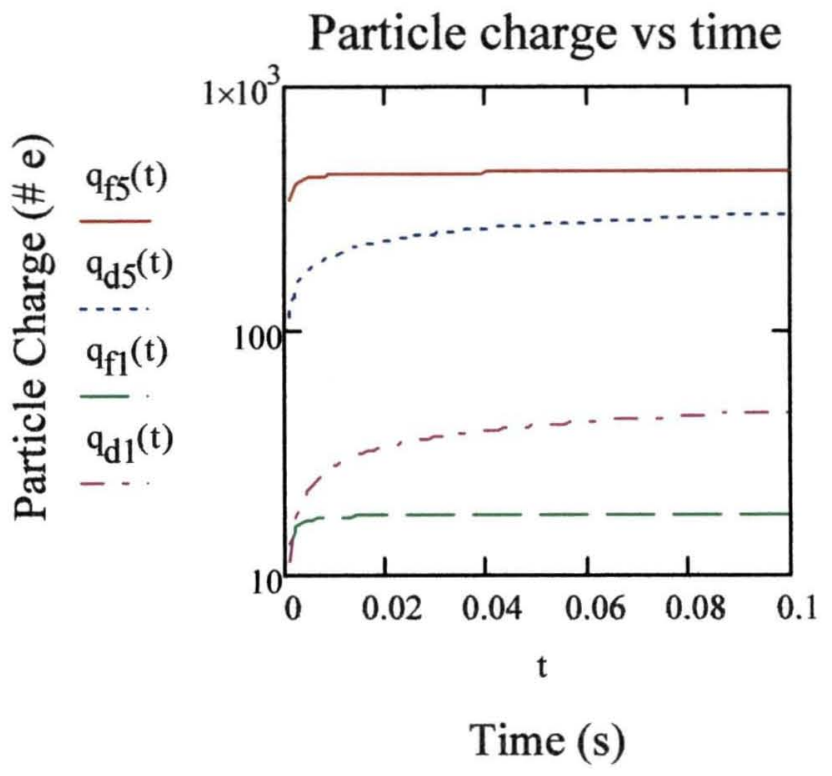

Fig. 4. Continuum regime field (Pauthenier) charge and diffusion charge for particles in $\mathrm{CO}_{2}$ at 9 mbars with $\mathrm{E}=0.35 \mathrm{kV} / \mathrm{cm}$ plotted vs. exposure time using (4) and (6). The upper two curves (solid-field, dotted-diffusion) are for a $5 \mu \mathrm{m}$ diameter particle, and the lower two (long dash-field, dot/dash-diffusion) are for a $1 \mu \mathrm{m}$ particle.

Once the dust particles are charged to a level $q$, they experience an $F=q E$ force which drives them to the collecting electrode. They drift towards the electrode with a terminal velocity (migration velocity) determined by the gas drag. In the Stoke's regime (Reynold's number less than one), the drag force is given by Stoke's law with the Cunningham slip correction factor $C_{c}$ required for small Knudsen numbers, i.e., when the particle size is not much 
greater than the gas molecule mean free path [17]. The migration velocity $w$ is given by [2],[4],[5],[17]

$$
w=C_{c} \frac{q E}{3 \pi \eta d}
$$

with

$$
C_{c}=1+K_{n}\left(\alpha+\beta e^{\frac{-\gamma}{K_{n}}}\right)
$$

where $\eta$ is the gas viscosity at that gas temperature, $d$ is the particle diameter, and $\alpha, \beta, \gamma$ are parameters that depend on the gas composition and particle shape, etc. For $\mathrm{CO}_{2}$ at STP $\eta=1.47 \times 10^{-5} \mathrm{~N} \cdot \mathrm{s} / \mathrm{m}^{2}$ and $\alpha=1.15, \beta=0.48$ and $\gamma=0.92$ for solid spherical particles in $\mathrm{CO}_{2}$ [12]. The Knudsen number $K_{n}=2 \lambda d d=8.5$ for $1-\mu \mathrm{m}$ diameter particles in the Mars atmosphere at $273 \mathrm{~K}$. The Cunningham correction factor for $K_{n}=8.5$ in $\mathrm{CO}_{2}$ is approximately 15 . The low gas pressure on Mars increases $w$ because of the Cunningham slip factor, but also decreases $w$ because $E$ and $q$ are limited to low values due to gas breakdown. For example, a1- $\mu$ m diameter particle in an ESP on Mars with $E=0.23 \mathrm{kV} / \mathrm{cm}$ with a roughly estimated charge of about 100 electrons would have a migration velocity $w=0.4 \mathrm{~m} / \mathrm{s}$, which is higher than the typical value on Earth of $0.1 \mathrm{~m} / \mathrm{s}$ for $1-\mu \mathrm{m}$ particles [4]. For $10-\mu \mathrm{m}$ particles with 2000 electrons, $K_{n}=0.85, C_{c}=2$, and $w=0.01 \mathrm{~m} / \mathrm{s}$. which is much lower than on Earth where $w=$ $1 \mathrm{~m} / \mathrm{s}$ for $10-\mu \mathrm{m}$ particles typically. Therefore a Martian ESP should work better than on Earth for $1 \mu \mathrm{m}$ particles due to the very low drag for small particles only, but much worse for 10- $\mu \mathrm{m}$ particles because of the low $E$.

The precipitator collection efficiency can be estimated using the Deutch-Anderson equation which applies to turbulent (high Reynold's number) flow in the ESP. The collection efficiency $C E$ is given by White [4], Oglesby et al [5], and Cross [2], as

$$
C E=1-e^{\left(\frac{A}{V} w\right)}
$$

where $A$ is the total collection electrode area, $V$ is the gas flow rate, and $w$ is the particle migration velocity.

\section{ELECTROSTATIC PRECIPITATOR I-V CURVES}

Three 30-cm long cylinders with inner diameters of 5.3$\mathrm{cm}, 7.1-\mathrm{cm}$, and $9.6-\mathrm{cm}, 0.32-\mathrm{cm}$ and $0.64-\mathrm{cm}$ rods, and a wire with $100-\mu \mathrm{m}$ diameter were used for the outer and inner electrodes of the precipitator. Numerous combinations were tested in a glass bell jar vacuum chamber (Fig. 5) evacuated to 9 mbars pressure. Current-voltage (I-V) curves were obtained for five geometries in a $95 \% \mathrm{CO}_{2}-5 \%$ humid air mixture to approximate the Martian atmospheric composition (Fig. 6). Negative corona, which is normally more stable than positive in atmospheric pressure air, yielded very steep I-V curves (not shown) with a narrow region of stability. This was most likely due to the smaller

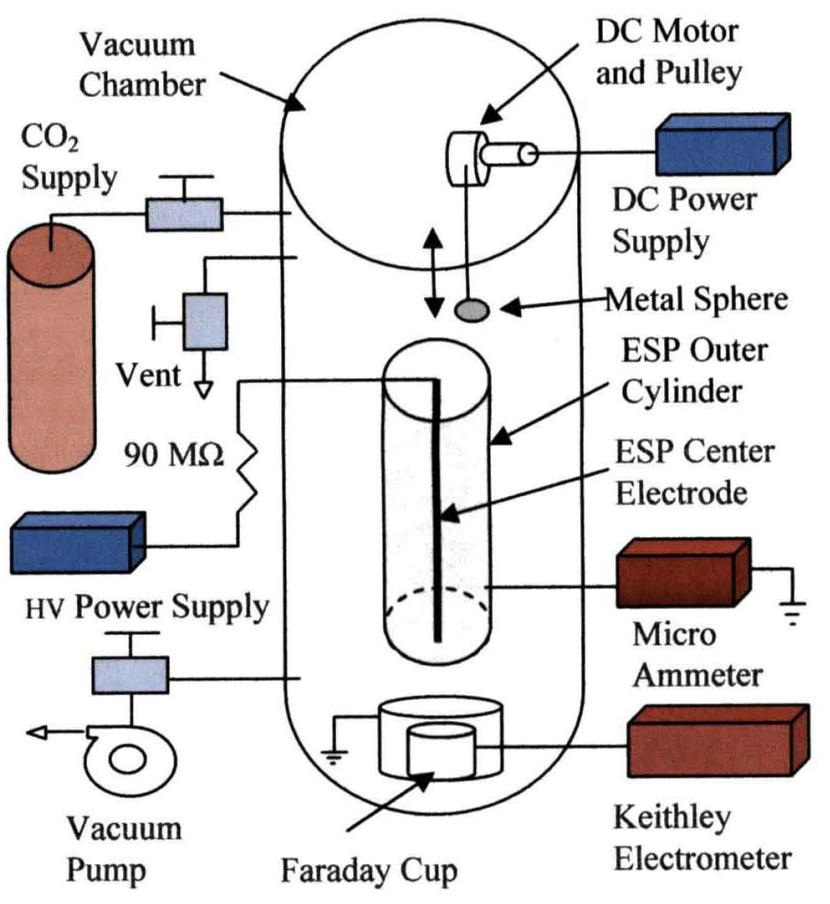

Fig. 5 Schematic diagram of the experimental apparatus.

\section{ESP I-V Curves}

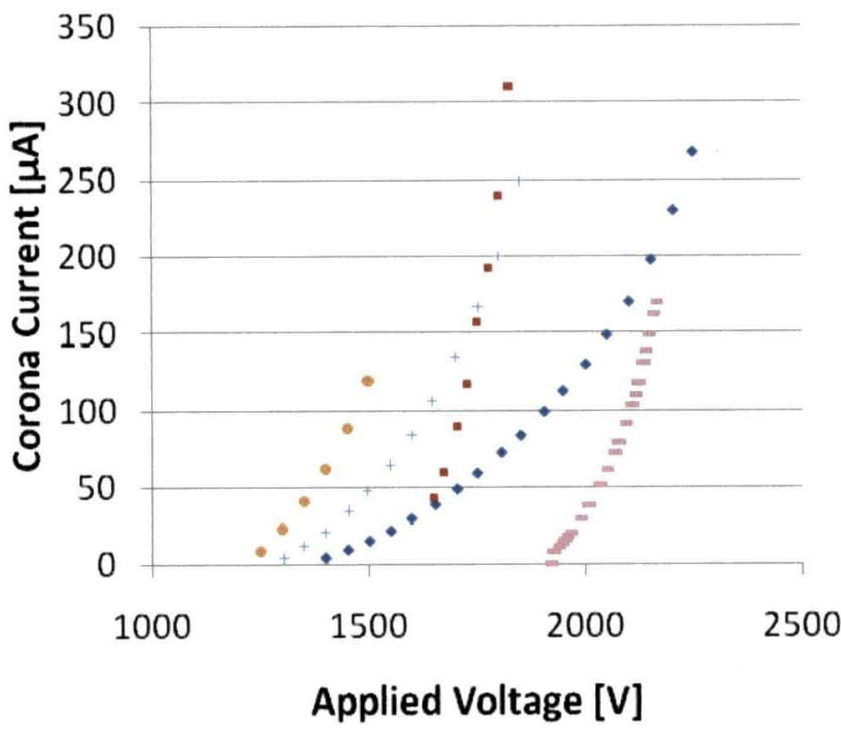

- 100 micron wire $9.6 \mathrm{~cm}$ diameter cylinder

+ 100 micron wire $7.1 \mathrm{~cm}$ diameter cylinder

- 100 micron wire $5.3 \mathrm{~cm}$ diameter cylinder

$-0.635 \mathrm{~cm}$ rod $9.6 \mathrm{~cm}$ diameter cylinder

- $0.315 \mathrm{~cm}$ rod $7.1 \mathrm{~cm}$ diameter cylinder

Fig. 6. I-V curves for five configurations of the precipitator. Data taken with clean electrodes and positive polarity at 9 mbars in $\mathrm{CO}_{2}$. 
electron attachment rates at the low gas pressure which results in more ionization and higher currents due to the $\sim 300 \times$ higher electron "mobilities" of the electrons [10]. More importantly, the negative corona only occurred on one location on the discharge wire, and turned into a streamer with a slight increase in voltage. In contrast, positive polarity resulted in a very uniform glow on the entire wire and had a much wider stable voltage range (Fig. 7 Left).
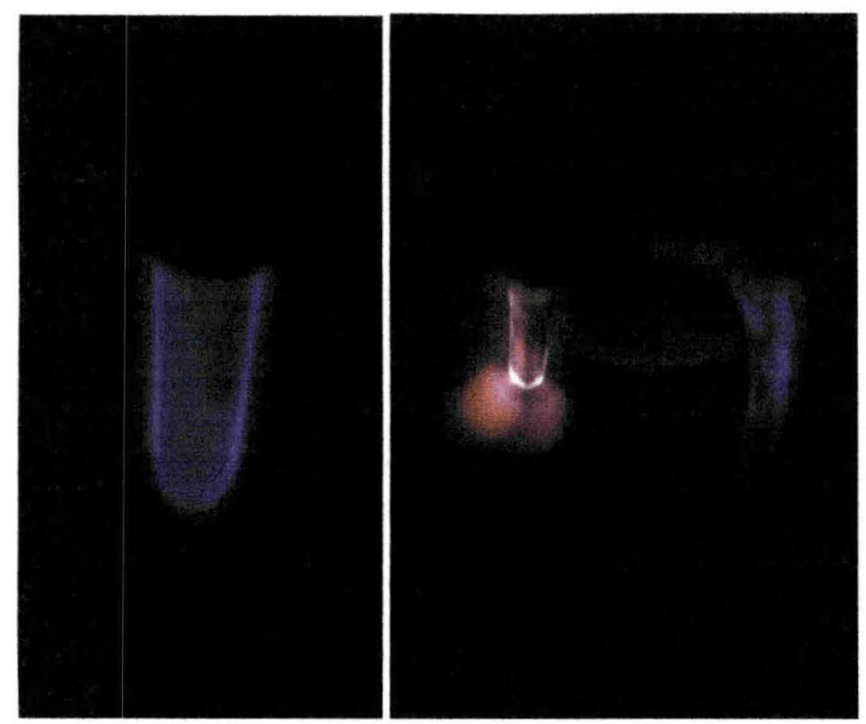

Fig. 7 Left) Stable positive corona at $2.2 \mathrm{kV}$ and $150 \mu \mathrm{A}$ on $0.64-\mathrm{cm}$ diameter rod inside $9.6-\mathrm{cm}$ diameter cylinder in $95 \% \mathrm{CO}_{2} / 5 \%$ humid air at 9 mbar taken using $50 \mathrm{~mm}$ lens at F16 with $20 \mathrm{~s}$ exposure.

Right) Same geometry just after transition from $200 \mu \mathrm{A}$ positive corona to unstable streamer discharge ( $\mathrm{F} 8,10 \mathrm{~s})$. Two stationary pink streamers are visible below the rod, as well as the recorded "dancing" motion of a dynamic blue streamer from the rod to the inner cylinder.

Because a stable, uniform corona discharge is required for effective particle charging, only positive polarity was used. In addition, a high voltage, 10-W, 90-M $\Omega$ resistor was added in series with the high voltage power supply to help stabilize the discharge. Using the I-V curves in Fig. 6, onset voltages for corona and streamer "breakdown" were determined. The data for the $0.32-\mathrm{cm}$ and $0.64-\mathrm{cm}$ rods emphasizes how different the corona discharge is at low pressures. In contrast to the atmospheric pressure case, a stable corona discharge was obtained at low voltages using these large diameter rods and also for a larger 1.26-cm rod (not shown).

\section{CORONA CHARGING EXPERIMENTS}

Experiments with three large brass spheres were performed to test the charging performance of the precipitator geometry in the continuum regime where Pauthenier field charging theory clearly applies. Spheres with diameters of $0.47 \mathrm{~cm}, 0.95 \mathrm{~cm}$ and $1.27 \mathrm{~cm}$ were lowered halfway into the precipitator-away from distorted edge fields - with a thin insulating Teflon thread (Fig. 5). Then the corona discharge was turned on for 10 seconds using a DC high voltage supply. The voltage level was measured using a Keithley 1000: 1 divider probe and the ion current with a Fluke 87 DMM. The supply was then turned off and the charged sphere was lowered into a shielded Faraday cup connected through a coaxial vacuum feedthrough to a Keithley 6514 electrometer to obtain the value of the charge acquired by the sphere. The zero check on the electrometer was not deactivated until several seconds after the high voltage was turned off to prevent stray ions from the corona from influencing the reading. In addition, the reading was not recorded until the sphere was raised out of the faraday cup so that the charge on the thread was not recorded. The conductive sphere was discharged when it touched the Faraday cup, which enabled the test to be repeated by simply raising the sphere back to the charging position inside the precipitator.

Calculated field charging values for the brass spheres are $\sim 1000 \times$ higher than diffusion charging values, therefore diffusion charging can be neglected. Thus, theoretical values for field charging of the spheres were calculated using the electric field value halfway between the wire and cylinder in (5) to compare to the experimental values obtained. The top half of Table I contains the results for spheres of three different sizes in the 7-cm diameter cylinder with a $100-\mu \mathrm{m}$ wire and $0.31-\mathrm{cm}$ diameter rod. The bottom half of Table I has data for the 9.6- $\mathrm{cm}$ cylinder with a 100 $\mu \mathrm{m}$ wire and $0.635-\mathrm{cm}$ rod. The average of ten tests (charge measurements) and the resulting standard deviation is shown for each condition.

Some of the measured values differed more than expected, based on their standard deviation, from the theoretical predictions. Variations in the exposure time should not matter because saturation is reached in milliseconds (Fig. 4). The differences are most likely due to the actual electric field $E$ being different than calculated which changes the Pauthenier saturation charge value because it is proportional to $E$. Almost all of the results at large $E$ field values were smaller than theory, probably because the $E$ field pushes the hanging charged sphere toward the collecting electrode into a lower $E$ field region. This error should be worse for the smaller spheres because the gravitational force that tries to keep the ball hanging straight down is proportional to the sphere mass (radius cubed), whereas the Coulomb force depends on area (radius squared). However, this was not the case in our tests, smaller spheres were affected less than larger ones at high $E$.

If the sphere hangs closer to the discharge wire than the halfway position $(0.5 R)$ assumed in the theoretical calculation, then $E$ and the corresponding $\mathrm{Q}_{\mathrm{s}}$, would be higher than predicted [ $E$ increases as $\mathrm{r}$ decreases, from (1)]. Or the opposite could happen, resulting in a lower $E$ and $Q_{s}$. Another source of error is the presence of the metal sphere shortening the inter-electrode gap and thereby increasing $E$. This effect should be proportional to the sphere diameter. The calculation of the space charge effect on $E$ is dependent on knowing the ion mobility $b$. If the actual ion mobility differed from the theoretical value due to the low gas pressure or ion clusters forming, etc., then the ion density and resulting $E$ would be different. Low mobility ion clusters are known to form in atmospheric pressure humid 
air, and the gas mixture used in the experiments contained $5 \%$ humid room air, although it was at low pressure.

The ball charge data in $95 \% \mathrm{CO}_{2}-5 \%$ humid air at 9 mbars for the $9.6-\mathrm{cm}$ cylinder with $100-\mu \mathrm{m}$ wire is plotted in Figs. 8 and 9. Error bars are not shown on the plots because the standard deviations (Table I) are too small to display. In Fig. 8, the charge varies linearly with $E$ as predicted: a linear best fit line is shown for each data series. All the plots should extend through the origin as $E$ is decreased because there is no Pauthenier charging at zero $E$, and diffusion charging is predicted to be $1000 \times$ lower in this size range. However, the larger sphere sizes have an unexpected charge offset. Fig. 9 has a plot of charge vs. the ball diameter fit with a power law. At $E=0.11 \mathrm{kV} / \mathrm{cm}$, the charge varies with the square of the diameter, as predicted by Pauthenier theory. At the higher $E$ field, the plot varies approximately with the square of the diameter (the 1.8 power instead of 2).

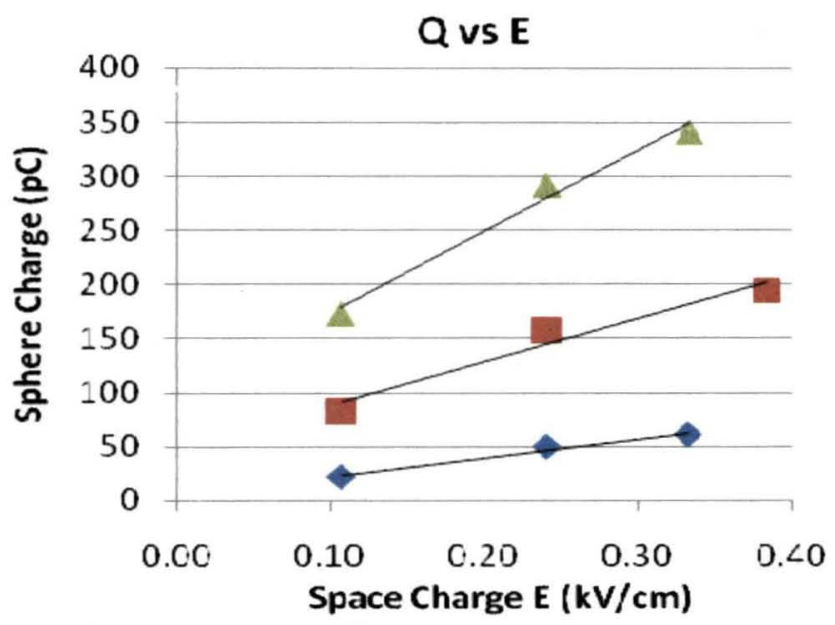

$\Delta 0.47 \mathrm{~cm}$ dia. $\quad 0.95 \mathrm{~cm} \mathrm{dia.} \Delta 1.27 \mathrm{cnd}$ a.

Fig. 8. Experimental values of the average charge on $0.47,0.95$ and $1.27 \mathrm{~cm}$ diameter brass spheres vs. $E$.

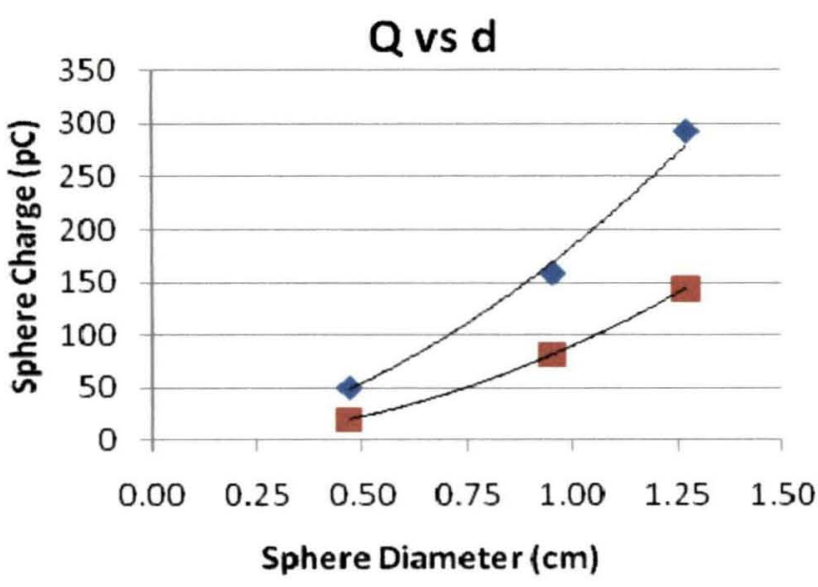

$\mathrm{E}=0.11 \mathrm{kV} / \mathrm{cm} \quad \forall=0.24 \mathrm{kV} / \mathrm{cm}$

Fig. 9. Experimental charge vs. sphere diameter for $E$ fields of 0.11 and $0.24 \mathrm{kV} / \mathrm{cm}$.

\section{CONCLUSIONS}

Human exploration of the planet Mars will require the utilization of local resources to reduce the mass, cost, and risk of those future missions. Among the most important resources are water and oxygen, which can be extracted from the Martian atmosphere, composed mostly of carbon dioxide. Before the Martian atmospheric gas can be processed for the production of these commodities, dust particles with diameters ranging from 2 to $10 \mu \mathrm{m}$ must be first removed. The current-voltage, I- $\mathrm{V}$, characteristic curves taken for the precipitator configurations at 9 mbars of pressure showed that a cylindrical collecting electrode either 7.1 or $9.6 \mathrm{~cm}$ in diameter with a concentric positive high voltage wire $100 \mu \mathrm{m}$ in diameter provides an adequate range of voltage and charging corona current. The charge acquired by brass spheres placed in several different geometries of our precipitator design agreed well with Pauthenier charging theory and showed that the precipitator produces the desired charge and collection electric field. This charging mechanism should also apply to the larger dust particles expected in the Martian atmosphere. Diffusion charging, the second charging mechanism applicable to the dust particles, has little effect on the large brass spheres.

\section{ACKNOWLEDGEMENT}

We thank Mr. Dana Greene for constructing numerous electrostatic precipitator electrodes and the test apparatus.

\section{REFERENCES}

[1] G. B. Sanders et al, "Results from the NASA Capability Roadmap Team for In-Situ Resource Utilization (ISRU)," in 2005 International Lunar Conference, Toronto, Canada.

[2] J. Cross, Electrostatics: Principles and Applications, London: IOP Publishing, 1987.

[3] H. L. Pang, P. Atten and J. L. Reboud, "Corona Discharge and Electrostatic Precipitation in Carbon Dioxide under Reduced Pressure Simulating Mars Atmosphere," IEEE Trans. on Industry Applications, vol. 45, no.1, pp. 50-58, 2009.

[4] H. J. White, Industrial Electrostatic Precipitation, Reading, MA Addison-Wesley, 1963.

[5] S. Olgesby and G. B. Nichols, Electrostatic Precipitation, New York,: Marcel Dekker, 1978.

[6] G. A. Landis, K. Herkenhoff, R. Greeley, S. Thompon, P. Whelley and the MER Athena Science Team, "Dust and Sand Deposition on the MER Solar Arrays as Viewed by the Microscopic Imager," Lunar and Planetary Science, vol. 37, p. 1937, 2006.

[7] J. S. Townsend, Electricity in Gases, New York: Oxford University Press, 1914.

[8] F. Paschen, Wied. Ann, vol. 37, p. 69, 1889.

[9] J. J. Thomson and G. P. Thomson, Conduction of Electricity through Gases, London: Cambridge University Press, 1933.

[10] J. Dutton, "A Survey of Electron Swarm Data," J. Physical Chemistry Reference Data, vol. 4, 1975.

[11] C. I. Calle. J. S. Clements, J. Willis and C. R. Buhler, "Paschen Breakdown Experiments in a Martian Atmosphere," NASA Technical Memorandum 2004-211535, pp.86-87, 2004.

[12] O. Schmid, M. B. Trueblood, N. Gregg, D. E. Hagen, P. D. Whitefield, "Sizing of Aerosol Particles in Gases other than Air using a Differential Mobility Analyzer," Aerosol and Science Technology, vol. 36, pp. 351-360, 2002. 
[13] K. L. Kaiser, Electromagnetic Compatibility Handbook, Boca Raton: CRC Press, pp. 10-97, 2005.

[14] A. F. Kovarik, "Mobility of Positive and Negative Ions in Gases at High Pressures," Proc. of Royal Society of London: Series A, vol. 86, no. 584, pp. 154-162, 1912.

[15] M. M. Pauthenier and M. Moreau-Hanot, "La Charge de Particules Spheriques dans un Champ Ionise," J. Phys. Radium Ser.,. vol. 73, pp. 500-613, 1932.

[16] P. Arendt and H. Kallman, "The Mechanism of Charging Mist Particles," Z. Physics vol. 35, pp. 421-441, 1926.

[17] W. C. Hinds, Aerosol Technology, New York: John Wiley, 1999.

[18] W. B. Smith and J. R. McDonald, "Development of a Theory for the Charging of Particles by Unipolar Ions," J. Aerosol Sci., vol. 7 pp. 151-156, 1976.
[19] B. Y. H. Liu and A. Kapadia, "Combined Field and Diffusion Charging of Aerosol Particles in the Continuum Regime," J. Aerosol Science, vol. 9, pp. 227-242, 1978.

[20] F. J. Romai and D. Y. H. Piu, "On the Combination Coefficient of Positive Ions with Ultra-Fine Neutral Particles in the Transition and Free Molecular Regimes," J. Aero. Sci. vol. 23, pp. 679-692, 1993.

[21] A. V. Filippov, "Charging of Aerosol in the Transition Regime," $J$. Aerosol Science, vol. 24, no. 4, pp. 424-436, 1993.

[22] W. H. Marlow and J. R. Brock, "Unipolar Charging of Small Unipolar Particles," J. Colloid Interface Science vol. 50, pp. 32-38, 1975.

[23] S. W. Davison and J. L. Gentry, "Difference in the Diffusion Charging of Dielectric and Conducting Ultra-Fine Aerosols," Aerosol Sci. Tech., vol. 4, pp. 157-163, 1985.

TABLE I

TOP HALF: BRASS SPHERE CHARGING EXPERIMENTS IN THE 7.1-CM DIAMETER ESP WITH 100- $\mu$ M WIRE AND 0.3175-CM ROD

BOTTOM HALF: 9.6-CM DIAMETER ESP WITH 100- $\mu$ M DIAMETER WIRE AND 0.635-CM DIAMETER ROD

\begin{tabular}{|c|c|c|c|c|c|c|c|c|c|}
\hline $\begin{array}{c}\text { Wire/rod } \\
\text { diameter } \\
{[\mu \mathrm{m}]} \\
\end{array}$ & $\begin{array}{c}\text { Sphere } \\
\text { diameter } \\
{[\mathrm{cm}]} \\
\end{array}$ & $\begin{array}{c}\text { Average } \\
\text { Applied } \\
\text { Voltage } \\
{[\mathrm{V}]} \\
\end{array}$ & $\begin{array}{c}\text { Ion } \\
\text { Current } \\
{[\mathbf{u A}]} \\
\end{array}$ & $\begin{array}{c}0.5 R \text { Ion } \\
\text { density } N_{o} \\
{\left[\# / \mathbf{m}^{3}\right]}\end{array}$ & $\begin{array}{c}\text { Space } Q \\
0.5 R \\
\text { E-field } \\
{[\mathrm{kV} / \mathrm{cm}]}\end{array}$ & $\begin{array}{l}\text { Theoretical } \\
\text { Pauthenier } \\
\text { Saturation } \\
\text { Charge [pC] }\end{array}$ & $\begin{array}{c}\text { Average } \\
\text { Measured } \\
\text { Charge } \\
\text { [pCl } \\
\end{array}$ & $\begin{array}{c}\text { Charge } \\
\text { Standard } \\
\text { Deviation } \\
{[p C]} \\
\end{array}$ & $\begin{array}{c}\text { Exp.-Theory } \\
\text { Percent } \\
\text { Difference } \\
{[\%]} \\
\end{array}$ \\
\hline 100 & 0.47 & 1696 & 126 & $8.9 \mathrm{E}+13$ & 0.34 & 63 & 66 & 6 & 4 \\
\hline 100 & 0.95 & 1318 & 5 & $8.7 E+12$ & 0.13 & 98 & 110 & 7 & 13 \\
\hline 100 & 0.95 & 1509 & 51 & $5.2 \mathrm{E}+13$ & 0.24 & 180 & 166 & 5 & -8 \\
\hline 100 & 0.95 & 1690 & 127 & $8.9 E+13$ & 0.35 & 262 & 225 & 7 & -14 \\
\hline 100 & 1.27 & 1433 & 32 & $3.8 \mathrm{E}+13$ & 0.20 & 269 & 261 & 7 & -3 \\
\hline 100 & 1.27 & 1577 & 79 & $6.8 \mathrm{E}+13$ & 0.28 & 379 & 323 & 9 & -15 \\
\hline 100 & 1.27 & 1674 & 124 & $8.8 \mathrm{E}+13$ & 0.34 & 460 & 362 & 9 & -21 \\
\hline 3175 & 0.95 & 1654 & 16 & $1.2 \mathrm{E}+13$ & 0.31 & 236 & 257 & 1 & 9 \\
\hline 3175 & 0.95 & 1687 & 45 & $3.0 \mathrm{E}+13$ & 0.35 & 265 & 262 & 1 & -1 \\
\hline 3175 & 0.95 & 1784 & 85 & $5.0 \mathrm{E}+13$ & 0.40 & 300 & 257 & 6 & -14 \\
\hline 100 & 0.47 & 1406 & 6 & $9.1 \mathrm{E}+12$ & 0.11 & 20 & 23 & 10 & 14 \\
\hline 100 & 0.47 & 1744 & 61 & $4.5 \mathrm{E}+13$ & 0.24 & 44 & 50 & 3 & 12 \\
\hline 100 & 0.47 & 1982 & 125 & $6.7 e+13$ & 0.33 & 61 & 61 & 9 & -1 \\
\hline 100 & 0.95 & 1434 & 5 & $8.4 \mathrm{E}+12$ & 0.11 & 81 & 83 & 2 & 3 \\
\hline 100 & 0.95 & 1771 & 60 & $4.4 \mathrm{E}+13$ & 0.24 & 182 & 158 & 4 & -13 \\
\hline 100 & 0.95 & 2093 & .171 & $7.9 \mathrm{E}+13$ & 0.38 & 291 & 195 & 7 & -33 \\
\hline 100 & 1.27 & 1418 & 5 & $8.5 \mathrm{E}+12$ & 0.11 & 144 & 173 & 6 & 21 \\
\hline 100 & 1.27 & 1761 & 60 & $4.5 \mathrm{E}+13$ & 0.24 & 324 & 292 & 6 & -10 \\
\hline 100 & 1.27 & 1980 & 126 & $6.7 \mathrm{E}+13$ & 0.33 & 449 & 342 & 7 & -24 \\
\hline 6350 & 0.47 & 1918 & 8 & $5.8 \mathrm{E}+12$ & 0.29 & 53 & 51 & 2 & -5 \\
\hline 6350 & 0.47 & 2075 & 91 & $4.6 \mathrm{E}+13$ & 0.40 & 74 & 66 & 2 & -11 \\
\hline 6350 & 0.47 & 2126 & 142 & $6.4 \mathrm{E}+13$ & 0.45 & 83 & 70 & 2 & -16 \\
\hline 6350 & 0.95 & 1926 & 9 & $6.2 \mathrm{E}+12$ & 0.29 & 220 & 249 & 5 & 13 \\
\hline 6350 & 0.95 & 2085 & 91 & $4.6 \mathrm{E}+13$ & 0.40 & 303 & 250 & 5 & -17 \\
\hline 6350 & 1.27 & 1965 & 12 & $8.0 \mathrm{E}+12$ & 0.30 & 404 & 435 & 6 & 8 \\
\hline 6350 & 1.27 & 2161 & 128 & $5.9 \mathrm{E}+13$ & 0.44 & 594 & 433 & 4 & -27 \\
\hline
\end{tabular}

